PROCEEDINGS OF THE

AMERICAN MATHEMATICAL SOCIETY

Volume 126, Number 1, January 1998, Pages 65-66

S 0002-9939(98)04134-3

\title{
FUSION IN THE CHARACTER TABLE
}

\author{
GABRIEL NAVARRO \\ (Communicated by Ronald M. Solomon)
}

\begin{abstract}
Suppose that $P$ is a Sylow $p$-subgroup of a finite $p$-solvable group $G$. If $g \in P$, then the number of $G$-conjugates of $g$ in $P$ can be read off from the character table of $G$.
\end{abstract}

One of the classical problems in character theory is to find what kind of group theoretical information the character table determines.

Theorem A. Let $P$ be a Sylow p-subgroup of a finite p-solvable group $G$. If $K$ is a conjugacy class of $G$, then $|K \cap P|$ can be read off from the character table of $G$.

Although, it is possible to find non-isomorphic non-p-solvable groups with the same character table, it does not seem easy to construct examples where the conclusion of Theorem A fails. In fact, we shall prove Theorem A by using some of the deep facts in Isaacs $\pi$-theory. We should say that it is not clear at all how to find a direct proof without it.

Proof of Theorem A. Let $K$ be a conjugacy class of a $\pi$-separable group $G$ and let $H$ be a Hall $\pi$-subgroup of $G$. We prove that the integer $|K \cap H|$ can be read off from the character table of $G$.

By a theorem of G. Higman ((8.21) of [3]), we know that the character table determines the set of primes that divide the common order of the elements in $K$. Therefore, we may assume that $K$ consists of $\pi$-elements (otherwise, $|K \cap H|$ is zero). By Higman's Theorem, let $K_{1}, \ldots, K_{h}$ be the conjugacy classes of $G$ consisting of $\pi$-elements, so that $K$ is one of them. Write $G^{0}=K_{1} \cup \ldots \cup K_{h}$, the set of $\pi$ elements of $G$. Also, let us denote by $\alpha^{0}$ the restriction of a class function $\alpha$ of $G$ to $G^{0}$. Among the functions $\chi^{0}$ for $\chi \in \operatorname{Irr}(G)$, we consider the subset $\mathrm{I}_{\pi}(G)$ consisting of those functions that cannot be written in the form $\nu^{0}+\mu^{0}$ for nonzero characters $\nu$ and $\mu$ of $G$. By Theorem A of [1], the set $\mathrm{I}_{\pi}(G)$ forms a basis for the vector space $\operatorname{cf}\left(G^{0}\right)$ of class functions on $G^{0}$. Hence, notice that, by using the character table, we may find $\left\{\chi_{1}, \ldots, \chi_{h}\right\} \subseteq \operatorname{Irr}(G)$ such that $I_{\pi}(G)=\left\{\left(\chi_{1}\right)^{0}, \ldots,\left(\chi_{h}\right)^{0}\right\}$ (given $\chi \in \operatorname{Irr}(G)$, since $\chi(1)$ is finite, there are only a finite number of characters $\nu, \mu$ to check the equation $\left.\chi^{0}=\nu^{0}+\mu^{0}\right)$. We may certainly assume that $\chi_{1}=1_{G}$.

Now, by Corollary (2.5) of [2], we have that $1_{H}$ is a 'Fong character' associated to $\left(1_{G}\right)^{0}$ and therefore,

$$
\left[\left(\chi_{i}\right)_{H}, 1_{H}\right]=0 \quad \text { for } i \geq 2 .
$$

Received by the editors August 5, 1996.

1991 Mathematics Subject Classification. Primary 20C15.

Research partially supported by DGICYT.

(C) 1998 American Mathematical Society 
If $x_{j} \in K_{j}$, then

$$
\left[\left(\chi_{i}\right)_{H}, 1_{H}\right]=\frac{1}{|H|} \sum_{j=1}^{h}\left|K_{j} \cap H\right| \chi_{i}\left(x_{j}\right)
$$

and thus, we may write

$$
\left(\begin{array}{c}
|H| \\
0 \\
\vdots \\
0
\end{array}\right)=\left(\begin{array}{ccc}
\chi_{1}\left(x_{1}\right) & \cdots & \chi_{1}\left(x_{h}\right) \\
\vdots & \ddots & \vdots \\
\chi_{h}\left(x_{1}\right) & \cdots & \chi_{h}\left(x_{h}\right)
\end{array}\right)\left(\begin{array}{c}
\left|K_{1} \cap H\right| \\
\vdots \\
\left|K_{h} \cap H\right|
\end{array}\right) .
$$

By Theorem A of [1], we know that the matrix

$$
\left(\begin{array}{ccc}
\chi_{1}\left(x_{1}\right) & \cdots & \chi_{1}\left(x_{h}\right) \\
\vdots & \ddots & \vdots \\
\chi_{h}\left(x_{1}\right) & \cdots & \chi_{h}\left(x_{h}\right)
\end{array}\right)
$$

is invertible. Since this matrix and $|H|=|G|_{\pi}$ are clearly determined by the character table, we have that

$$
\left(\begin{array}{c}
\left|K_{1} \cap H\right| \\
\vdots \\
\left|K_{h} \cap H\right|
\end{array}\right)
$$

is determined by the character table.

Although, in general, it is not clear how to calculate permutation characters from the character table, we may prove the following result.

Corollary B. Let $P$ be a Sylow p-subgroup of a finite p-solvable group $G$. Then the permutation character $\left(1_{P}\right)^{G}$ can be read off from the character table of $G$.

Proof. Again, we assume that $H$ is a Hall $\pi$-subgroup of a $\pi$-separable group $G$ and we determine $\left(1_{H}\right)^{G}$ from the character table of $G$. If $\chi \in \operatorname{Irr}(G)$, it is enough to show how to calculate $\left[\chi,\left(1_{H}\right)^{G}\right]$ from the character table of $G$. If $K_{1}, \ldots, K_{h}$ are the conjugacy classes of $G$ consisting of $\pi$-elements and $x_{j} \in K_{j}$, we have that

$$
\left[\chi,\left(1_{H}\right)^{G}\right]=\left[\chi_{H}, 1_{H}\right]=\frac{1}{|H|} \sum_{j=1}^{h}\left|K_{j} \cap H\right| \chi_{i}\left(x_{j}\right) .
$$

The result now follows from Theorem A.

\section{REFERENCES}

1. M. Isaacs, Characters of $\pi$-separable groups, J. Algebra, 86, (1984), 98-128. MR 85h:20012

2. M. Isaacs, Fong characters in $\pi$-separable groups, J. Algebra 99, (1986), 89-107. MR 87e:20015

3. M. Isaacs, Character Theory of Finite Groups, New York, Dover, 1994.

Departament d'Algebra, Facultat de Matematiques, Universitat de Valencia, 46100 Burjassot, Valencia, Spain

E-mail address: gabriel@uv.es 